Revue d'histoire de l'Amérique française

RAS REVUE D.HISTOIRE DE L'AMÉRIQUE FRANÇAISE

\title{
Recensement de la Seigneurie d'Eschambault (1688)
}

Volume 9, numéro 3, décembre 1955

URI : https://id.erudit.org/iderudit/301729ar

DOI : https://doi.org/10.7202/301729ar

Aller au sommaire du numéro

\section{Éditeur(s)}

Institut d'histoire de l'Amérique française

\section{ISSN}

0035-2357 (imprimé)

1492-1383 (numérique)

Découvrir la revue

Citer ce document

(1955). Recensement de la Seigneurie d'Eschambault (1688). Revue d'histoire de l'Amérique française, 9(3), 439-442. https://doi.org/10.7202/301729ar d'utilisation que vous pouvez consulter en ligne.

https://apropos.erudit.org/fr/usagers/politique-dutilisation/ 


\title{
DOCUMENTS INEDITS
}

\author{
RECENSEMENT DE LA SEIGNEURIE D'ESCHAMBAULT * \\ (1688)
}

Parmi quelques papiers de famille qui m'ont été remis il y a une trentaine d'années, par un cousin de la ville de Québec, Alphonse d'Eschambault, se trouve un document de portée plus générale. Il s'agit d'un recensement de la Seigneurie d'Eschambault, pièce originale, portant la date du 20 mai, 1688. Le document est de la main du Seigneur d'Eschambault et même si le temps a quelque peu effacé certains passages, le texte en est encore lisible. Nous croyons que les habitants actuels de Deschambault, tout comme les lecteurs de la Revue seront heureux de prendre connaissance de ce parchemin que les ans ont respecté.

$$
\begin{aligned}
& \text { Antoine d'EschambaulT, ptre, } \\
& \text { Saint-Boniface, Manitoba }
\end{aligned}
$$

Recensement de la Seigneurie et terres de deschambault que rend par devant Monseigneur l'Intandant Jacques Alexis Fleury Seigneur du dit lieu en conséquence de ... ordres en datte du... avril...

\section{Premièrement.}

Jacques Aléxis Fleury, Seigneur de la terre agé 48 ans 6 mois Marguerite de Chavigny sa femme en secondes noces agée de 49 ans 3. enfans de la ditte de Chavigny et de feu Thomas douaire, son premier mari.

Premier est Dorothée douaire et de présent en france agée de 36 ans 5 mois

Jacques douaire non marié et dans le pais agé de 30 ans 4 mois Louise douaire mariée aussi dans ce pais agée de 26 ans 5 mois

* Ce document nous a été gracieusement fourni par M. l'abbé Antoine d'Eschambault, de la Société historique de Saint-Boniface, Man. 
Augustin douaire non marié et aussi dans la pais, 25 ans 9 mois Enfants procrées du dit de Fleury et de Chavigny en second menage.

Premier est Jacques Aléxis Fleury en pension au Seminaire, agé de 19 ans 9 mois. Charles de Fleury agé de 17 ans 8 mois. Joseph de Fleury agé de 16 ans 2 mois. Louis de Fleury agé de 14 ans, 2 mois. Pierre de Fleury, agé de 11 ans 2 mois. Charlotte de Fleury agée de 10 ans, 4 mois. Simon de Fleury, agé de 6 ans. et les six cy dessus au Logis du sieur Deschambault. Ses domestiques, serviteurs et servantes. Premièrement est Pierre Fauscher engagé et enfuy (?) au monreal agé de 19 ans. Jean briere aussi engagé age 14 ans

Marie Codet servante engagée agée 18 ans

Ses bestiaux à cornes consistent en

six grands boeufs de charue agés de -2 de 8 ans

2 de 6

2 de 4

sept toreaux

2 agé de 3 ans
2 de 2

3 de cinq ans

sept meres vaches depuis 8 ans jusques a ans, deux genisse agée chacune de 2 ans. Une autre genisse agée de un an. Cinq veaux de l'an-4 masles et une génisse. six grands cochons savoir: 4 males

2 femelles pleines.

Terres labourables que fait exploiter le dit sieur dans son domaine seigneurial sur la dite Seigneurie ou actuellement il reside avec toutte sa famille ci dessus cottez

Nombre d'arpans ou environ à la charue

\section{premiermt}

Ceux qui sont chargez de bled forment autres chargez de poixs aussi à la charue environ 10 arpans autres aussi à la charue et laisse en guerets nombre de ceux qui ne sont encore a la charue et seulement à La pioche

Ceux qui sont ensemmencez

3 arpans de bled froment

2 arpans garny de poixs

2 arpans garny d'avoine

5 autres demeurez faulte dhomes

Nombre d'arpans de terre en prairie portant foing à fauscher ou autres à pasturer 
Ceux destinez à faire foing 16 arpans (mot illisible) cassé 6 autres (mot illisible)

Ceux destinez au pasturage 15 autres arpans environ Liste des habitants du dit lieu deschambault

Le premier est

Jullien Sanson, seul mais pensant (mot effacés) ... agé de 55 ans. Son habitation contient bien sept arpans de terre propre à recevoir la semmance et dont une bonne partie est chargez de bled forment, poixs, bled dinde avec graines nécessaires.

Le second est

Anthoine bessiere agé de 35 à 40 ans ou environ, marié depuis 2 années. Sa femme est Janne Crousteau agée de 18 à 20 ans et enceinte (mot illisible). Son habitation contient au environ 3 arpans de terre nette \& propre \& ensemmancé et un arpan et demy de bois abatus ou il travaille actuellement à le nettoyer et l'ensemmancer.

\section{Le troiziesme est}

françois Naust veuf depuis trois mois avec quatre enfans. Il est agé de 35 ans

Le premier est un garcon appellé françois Naust agé de 9 ans Le second est une fille appellée Genevieve agée de 6 ans Le troisième est une fille appellée Marguerite agée de 4 ans La quatrième est un garçon appellé Jan Baptiste Naust agé de 2 ans.

Son habitation contient environ 2 arpans ... (illisible) ... ensemmancée de bled froment et autres grains et environ cinq arpans de bois ou il travaille actuellement à nettoyer et à se bastir (quelques mots illisibles)

Nombre des bestiaux..

premièrement

meres vasches
veaux
cochons

veaux

Le quatriene est

Pierre Grolleau agé environ 45 ans et... mois.

Sa femme s'appelle Geneviève La Berge agée de 24 ans

Il a un enfan garçon appellé Jan Baptiste agé de... ans et sa femme enceinte. 
Son habitation consiste et contient au environ 3 arpans et demy de terre defrischée et une partie ensemmancée ou il travaille encore actuellement pour l'accroissement et augmenter son champ et a s'y batir... pour... et resider pour l'avenir. 2 mere Vasche

2 toreau de deux ans

une torre aussi de deux ans

2 cochons

Il $\mathrm{y}$ en a quatre autres familles qui ont reteint chacune une terre et qui vont incessamment y travailler que je ne cotte pas ( " cotte pas " est rayé et remplacé par quelques mots illisibles), attendu qu'elles n'y demeurent pas encome Comme

Jan La motte non marié agé de 22 ans qui a bien 2 arpans de bois abattus et buschez depuis un hyver

Autre Matthurin Chayé agé de 50 ans marié et qui a 5 enfans et sa femme

Autre Matthurin Chayé fils agé environ 22 ans.

fait a Deschambault ce 20e May 1688

Des Fleury Deschambault. 